\title{
Subclasses of $\lambda$-bi-pseudo-starlike functions with respect to symmetric points based on shell-like curves
}

\section{H. ÖZLEM GÜNEY ${ }^{1}$}

G. Murugusundaramoorthy ${ }^{2}$

K. VIJAYA ${ }^{2}$

1 Dicle University, Faculty of Science, Department of Mathematics, Diyarbakur, Turkey.

ozlemg@dicle.edu.tr

2 School of Advanced Sciences, Vellore Institute of Technology, Vellore-632014, India.

gmsmoorthy@yahoo.com;

kvijaya@vit.ac.in

\section{ABSTRACT}

In this paper we define the subclass $\mathcal{P} \mathcal{S}_{s, \Sigma}^{\lambda}(\alpha, \tilde{p}(z))$ of the class $\Sigma$ of bi-univalent functions defined in the unit disk, called $\lambda$-bi-pseudo-starlike, with respect to symmetric points, related to shell-like curves connected with Fibonacci numbers. We determine the initial Taylor-Maclaurin coefficients $\left|a_{2}\right|$ and $\left|a_{3}\right|$ for functions $f \in \mathcal{P} \mathcal{S}_{\mathcal{L}_{s, \Sigma}^{\lambda}}^{\lambda}(\alpha, \tilde{p}(z))$. Further we determine the Fekete-Szegö result for the function class $\mathcal{P} \mathcal{L}_{s, \Sigma}^{\lambda}(\alpha, \tilde{p}(z))$ and for the special cases $\alpha=0, \alpha=1$ and $\tau=-0.618$ we state corollaries improving the initial TaylorMaclaurin coefficients $\left|a_{2}\right|$ and $\left|a_{3}\right|$.

\section{RESUMEN}

En este artículo definimos la subclase $\mathcal{P} \mathcal{S}_{s, \Sigma}^{\lambda}(\alpha, \tilde{p}(z))$ de la clase $\Sigma$ de funciones bi-univalentes definidas en el disco unitario, llamadas $\lambda$-bi-pseudo-estrelladas, con respecto a puntos simétricos, relacionadas a curvas espirales en conexión con números de Fibonacci. Determinamos los coeficientes iniciales de Taylor-Maclaurin $\left|a_{2}\right|$ y $\left|a_{3}\right|$ para funciones $f \in$ $\mathcal{P} \mathcal{L}_{s, \Sigma}^{\lambda}(\alpha, \tilde{p}(z))$. Más aún determinamos el resultado de Fekete-Szegö para la clase de funciones $\mathcal{P} \mathcal{S L}_{s, \Sigma}^{\lambda}(\alpha, \tilde{p}(z))$ y para los casos especiales $\alpha=0, \alpha=1$ y $\tau=-0.618$ enunciamos corolarios mejorando los coeficientes iniciales de Taylor-Maclaurin $\left|a_{2}\right|$ y $\left|a_{3}\right|$.

Keywords and Phrases: Analytic functions, bi-univalent, shell-like curve, Fibonacci numbers, starlike functions.

2020 AMS Mathematics Subject Classification: 30C45, 30C50.

\section{(cC) BY-NC}

Accepted: 05 June, 2021

Received: 04 November, 2020
(C)2021 H. Özlem Güney et al. This open access article is licensed under a Creative Commons Attribution-NonCommercial 4.0 International License. 


\section{Introduction}

Let $\mathcal{A}$ denote the class of functions $f$ which are analytic in the open unit disk $\mathbb{U}=\{z: z \in \mathbb{C}$ and $|z|<1\}$. Also let $\mathcal{S}$ denote the class of functions in $\mathcal{A}$ which are univalent in $\mathbb{U}$ and normalized by the conditions $f(0)=f^{\prime}(0)-1=0$ and are of the form:

$$
f(z)=z+\sum_{n=2}^{\infty} a_{n} z^{n} .
$$

The Koebe one quarter theorem [4] ensures that the image of $\mathbb{U}$ under every univalent function $f \in \mathcal{A}$ contains a disk of radius $\frac{1}{4}$. Thus every univalent function $f$ has an inverse $f^{-1}$ satisfying

$$
f^{-1}(f(z))=z,(z \in \mathbb{U}) \text { and } f\left(f^{-1}(w)\right)=w \quad\left(|w|<r_{0}(f), r_{0}(f) \geq \frac{1}{4}\right) .
$$

A function $f \in \mathcal{A}$ is said to be bi-univalent in $\mathbb{U}$ if both $f$ and $f^{-1}$ are univalent in $\mathbb{U}$. Let $\Sigma$ denote the class of bi-univalent functions defined in the unit disk $\mathbb{U}$. Since $f \in \Sigma$ has the Maclaurin series given by (1.1), a computation shows that its inverse $g=f^{-1}$ has the expansion

$$
g(w)=f^{-1}(w)=w-a_{2} w^{2}+\left(2 a_{2}^{2}-a_{3}\right) w^{3}+\cdots .
$$

We notice that the class $\Sigma$ is not empty. For example, the functions $z, \frac{z}{1-z},-\log (1-z)$ and $\frac{1}{2} \log \frac{1+z}{1-z}$ are members of $\Sigma$. However, the Koebe function is not a member of $\Sigma$. In fact, Srivastava et al. [15] have actually revived the study of analytic and bi-univalent functions in recent years, it was followed by such works as those by (see $[2,3,9,15,16,17])$.

An analytic function $f$ is subordinate to an analytic function $F$ in $\mathbb{U}$, written as $f \prec F(z \in \mathbb{U})$, provided there is an analytic function $\omega$ defined on $\mathbb{U}$ with $\omega(0)=0$ and $|\omega(z)|<1$ satisfying $f(z)=F(\omega(z))$. It follows from Schwarz Lemma that

$$
f(z) \prec F(z) \quad \Longleftrightarrow \quad f(0)=F(0) \text { and } f(\mathbb{U}) \subset F(\mathbb{U}), z \in \mathbb{U}
$$

(for details see $[4,8])$. We recall important subclasses of $\mathcal{S}$ in geometric function theory such that if $f \in \mathcal{A}$ and

$$
\frac{z f^{\prime}(z)}{f(z)} \prec p(z) \quad \text { and } \quad 1+\frac{z f^{\prime \prime}(z)}{f^{\prime}(z)} \prec p(z)
$$

where $p(z)=\frac{1+z}{1-z}$, then we say that $f$ is starlike and convex, respectively. These functions form known classes denoted by $\mathcal{S}^{*}$ and $\mathcal{C}$, respectively. Recently, in [14], Sokół introduced the class $\mathcal{S} \mathcal{L}$ of shell-like functions as the set of functions $f \in \mathcal{A}$ which is described in the following definition:

Definition 1.1. The function $f \in \mathcal{A}$ belongs to the class $\mathcal{S L}$ if it satisfies the condition that

$$
\frac{z f^{\prime}(z)}{f(z)} \prec \tilde{p}(z)
$$

with

$$
\tilde{p}(z)=\frac{1+\tau^{2} z^{2}}{1-\tau z-\tau^{2} z^{2}},
$$

where $\tau=(1-\sqrt{5}) / 2 \approx-0.618$. 
It should be observed $\mathcal{S} \mathcal{L}$ is a subclass of the starlike functions $\mathcal{S}^{*}$.

The function $\tilde{p}$ is not univalent in $\mathbb{U}$, but it is univalent in the disc $|z|<(3-\sqrt{5}) / 2 \approx 0.38$. For example, $\tilde{p}(0)=\tilde{p}(-1 / 2 \tau)=1$ and $\tilde{p}\left(e^{\mp i \arccos (1 / 4)}\right)=\sqrt{5} / 5$, and it may also be noticed that

$$
\frac{1}{|\tau|}=\frac{|\tau|}{1-|\tau|}
$$

which shows that the number $|\tau|$ divides $[0,1]$ such that it fulfils the golden section. The image of the unit circle $|z|=1$ under $\tilde{p}$ is a curve described by the equation given by

$$
(10 x-\sqrt{5}) y^{2}=(\sqrt{5}-2 x)(\sqrt{5} x-1)^{2}
$$

which is translated and revolved trisectrix of Maclaurin. The curve $\tilde{p}\left(r e^{i t}\right)$ is a closed curve without any loops for $0<r \leq r_{0}=(3-\sqrt{5}) / 2 \approx 0.38$. For $r_{0}<r<1$, it has a loop, and for $r=1$, it has a vertical asymptote. Since $\tau$ satisfies the equation $\tau^{2}=1+\tau$, this expression can be used to obtain higher powers $\tau^{n}$ as a linear function of lower powers, which in turn can be decomposed all the way down to a linear combination of $\tau$ and 1 . The resulting recurrence relationships yield Fibonacci numbers $u_{n}$ :

$$
\tau^{n}=u_{n} \tau+u_{n-1}
$$

In [11] Raina and Sokół showed that

$$
\begin{aligned}
\tilde{p}(z) & =\frac{1+\tau^{2} z^{2}}{1-\tau z-\tau^{2} z^{2}}=\left(t+\frac{1}{t}\right) \frac{t}{1-t-t^{2}} \\
& =\frac{1}{\sqrt{5}}\left(t+\frac{1}{t}\right)\left(\frac{1}{1-(1-\tau) t}-\frac{1}{1-\tau t}\right) \\
& =\left(t+\frac{1}{t}\right) \sum_{n=1}^{\infty} u_{n} t^{n}=1+\sum_{n=1}^{\infty}\left(u_{n-1}+u_{n+1}\right) \tau^{n} z^{n},
\end{aligned}
$$

where

$$
u_{n}=\frac{(1-\tau)^{n}-\tau^{n}}{\sqrt{5}}, \quad \tau=\frac{1-\sqrt{5}}{2}, \quad t=\tau z \quad(n=1,2, \ldots) .
$$

This shows that the relevant connection of $\tilde{p}$ with the sequence of Fibonacci numbers $u_{n}$, such that $u_{0}=0, u_{1}=1, u_{n+2}=u_{n}+u_{n+1}$ for $n=0,1,2, \cdots$. And they got

$$
\begin{aligned}
\tilde{p}(z) & =1+\sum_{n=1}^{\infty} \tilde{p}_{n} z^{n} \\
& =1+\left(u_{0}+u_{2}\right) \tau z+\left(u_{1}+u_{3}\right) \tau^{2} z^{2}+\sum_{n=3}^{\infty}\left(u_{n-3}+u_{n-2}+u_{n-1}+u_{n}\right) \tau^{n} z^{n} \\
& =1+\tau z+3 \tau^{2} z^{2}+4 \tau^{3} z^{3}+7 \tau^{4} z^{4}+11 \tau^{5} z^{5}+\cdots
\end{aligned}
$$

Let $\mathcal{P}(\beta), 0 \leq \beta<1$, denote the class of analytic functions $p$ in $\mathbb{U}$ with $p(0)=1$ and $\operatorname{Re}\{p(z)\}>\beta$. Especially, we will use $\mathcal{P}$ instead of $\mathcal{P}(0)$. 
Theorem 1.2. [6] The function $\tilde{p}(z)=\frac{1+\tau^{2} z^{2}}{1-\tau z-\tau^{2} z^{2}}$ belongs to the class $\mathcal{P}(\beta)$ with $\beta=\sqrt{5} / 10 \approx$ 0.2236 .

Now we give the following lemma which will use in proving.

Lemma 1.3. [10] Let $p \in \mathcal{P}$ with $p(z)=1+c_{1} z+c_{2} z^{2}+\cdots$, then

$$
\left|c_{n}\right| \leq 2, \quad \text { for } \quad n \geq 1
$$

\section{Bi-Univalent function class $\mathcal{P S}_{\mathcal{L}_{s, \Sigma}^{\lambda}}^{(\alpha, \tilde{p}(z))}$}

In this section, we introduce a new subclass of $\Sigma$ associated with shell-like functions connected with Fibonacci numbers and obtain the initial Taylor coefficients $\left|a_{2}\right|$ and $\left|a_{3}\right|$ for the function class by subordination.

Firstly, let $p(z)=1+p_{1} z+p_{2} z^{2}+\cdots$, and $p \prec \tilde{p}$. Then there exists an analytic function $u$ such that $|u(z)|<1$ in $\mathbb{U}$ and $p(z)=\tilde{p}(u(z))$. Therefore, the function

$$
h(z)=\frac{1+u(z)}{1-u(z)}=1+c_{1} z+c_{2} z^{2}+\cdots
$$

is in the class $\mathcal{P}$. It follows that

$$
u(z)=\frac{c_{1} z}{2}+\left(c_{2}-\frac{c_{1}^{2}}{2}\right) \frac{z^{2}}{2}+\left(c_{3}-c_{1} c_{2}+\frac{c_{1}^{3}}{4}\right) \frac{z^{3}}{2}+\cdots
$$

and

$$
\begin{aligned}
\tilde{p}(u(z))= & 1+\frac{\tilde{p}_{1} c_{1} z}{2}+\left\{\frac{1}{2}\left(c_{2}-\frac{c_{1}^{2}}{2}\right) \tilde{p}_{1}+\frac{c_{1}^{2}}{4} \tilde{p}_{2}\right\} z^{2} \\
& +\left\{\frac{1}{2}\left(c_{3}-c_{1} c_{2}+\frac{c_{1}^{3}}{4}\right) \tilde{p}_{1}+\frac{1}{2} c_{1}\left(c_{2}-\frac{c_{1}^{2}}{2}\right) \tilde{p}_{2}+\frac{c_{1}^{3}}{8} \tilde{p}_{3}\right\} z^{3}+\cdots
\end{aligned}
$$

And similarly, there exists an analytic function $v$ such that $|v(w)|<1$ in $\mathbb{U}$ and $p(w)=\tilde{p}(v(w))$. Therefore, the function

$$
k(w)=\frac{1+v(w)}{1-v(w)}=1+d_{1} w+d_{2} w^{2}+\cdots
$$

is in the class $\mathcal{P}(0)$. It follows that

$$
v(w)=\frac{d_{1} w}{2}+\left(d_{2}-\frac{d_{1}^{2}}{2}\right) \frac{w^{2}}{2}+\left(d_{3}-d_{1} d_{2}+\frac{d_{1}^{3}}{4}\right) \frac{w^{3}}{2}+\cdots
$$

and

$$
\begin{aligned}
\tilde{p}(v(w))= & 1+\frac{\tilde{p}_{1} d_{1} w}{2}+\left\{\frac{1}{2}\left(d_{2}-\frac{d_{1}^{2}}{2}\right) \tilde{p}_{1}+\frac{d_{1}^{2}}{4} \tilde{p}_{2}\right\} w^{2} \\
& +\left\{\frac{1}{2}\left(d_{3}-d_{1} d_{2}+\frac{d_{1}^{3}}{4}\right) \tilde{p}_{1}+\frac{1}{2} d_{1}\left(d_{2}-\frac{d_{1}^{2}}{2}\right) \tilde{p}_{2}+\frac{d_{1}^{3}}{8} \tilde{p}_{3}\right\} w^{3}+\cdots
\end{aligned}
$$


The class $\mathcal{L}_{\lambda}(\alpha)$ of $\lambda$-pseudo-starlike functions of order $\alpha(0 \leq \alpha<1)$ were introduced and investigated by Babalola [1] whose geometric conditions satisfy

$$
\Re\left(\frac{z\left(f^{\prime}(z)\right)^{\lambda}}{f(z)}\right)>\alpha, \quad \lambda>0 .
$$

He showed that all pseudo-starlike functions are Bazilevič of type $\left(1-\frac{1}{\lambda}\right)$ order $\alpha^{\frac{1}{\lambda}}$ and univalent in open unit disk $\mathbb{U}$. If $\lambda=1$, we have the class of starlike functions of order $\alpha$, which in this context, are 1-pseudo-starlike functions of order $\alpha$. A function $f \in \mathcal{A}$ is starlike with respect to symmetric points in $\mathbb{U}$ if for every $r$ close to $1, r<1$ and every $z_{0}$ on $|z|=r$ the angular velocity of $f(z)$ about $f\left(-z_{0}\right)$ is positive at $z=z_{0}$ as $z$ traverses the circle $|z|=r$ in the positive direction. This class was introduced and studied by Sakaguchi [13] presented the class $\mathcal{S}_{s}^{*}$ of functions starlike with respect to symmetric points. This class consists of functions $f(z) \in \mathcal{S}$ satisfying the condition

$$
\Re\left(\frac{2 z f^{\prime}(z)}{f(z)-f(-z)}\right)>0, \quad z \in \mathbb{U} .
$$

Motivated by $\mathcal{S}_{s}^{*}$, Wang et al. [18] introduced the class $\mathcal{K}_{s}$ of functions convex with respect to symmetric points, which consists of functions $f(z) \in \mathcal{S}$ satisfying the condition

$$
\Re\left(\frac{2\left(z f^{\prime}(z)\right)^{\prime}}{(f(z)-f(-z))^{\prime}}\right)>0, \quad z \in \mathbb{U} .
$$

It is clear that, if $f(z) \in \mathcal{K}_{s}$, then $z f^{\prime}(z) \in \mathcal{S}_{s}^{*}$. For such a function $\phi$, Ravichandran [12] presented the following subclasses: A function $f \in A$ is in the class $\mathcal{S}_{s}^{*}(\phi)$ if

$$
\frac{2 z f^{\prime}(z)}{f(z)-f(-z)} \prec \phi(z), \quad z \in \mathbb{U}
$$

and in the class $\mathcal{K}_{s}(\phi)$ if

$$
\frac{2\left(z f^{\prime}(z)\right)^{\prime}}{(f(z)-f(-z))^{\prime}} \prec \phi(z) \quad z \in \mathbb{U} \text {. }
$$

Motivated by aforementioned works $[1,13,12,18]$ and recent study of Sokól [14] (also see [11]), in this paper we define the following new subclass $f \in \mathcal{P S}_{\mathcal{L}_{s, \Sigma}^{\lambda}}^{\lambda}(\tilde{p}(z))$ of $\Sigma$ named as $\lambda$-bi-pseudostarlike functions with respect to symmetric points, related to shell-like curves connected with Fibonacci numbers, and determine the initial Taylor-Maclaurin coefficients $\left|a_{2}\right|$ and $\left|a_{3}\right|$. Further we determine the Fekete-Szegö result for the function class $\mathcal{P S}_{\mathcal{L}_{s, \Sigma}^{\lambda}}^{\lambda}(\tilde{p}(z))$ and the special cases are stated as corollaries which are new and have not been studied so far.

Definition 2.1. For $0 \leq \alpha \leq 1 ; \lambda>0 ; \lambda \neq \frac{1}{3}$, a function $f \in \Sigma$ of the form (1.1) is said to be in the class $\mathcal{P S}_{\mathcal{L}_{s, \Sigma}^{\lambda}}^{\lambda}(\alpha, \tilde{p}(z))$ if the following subordination hold:

$$
\left(\frac{2 z\left(f^{\prime}(z)\right)^{\lambda}}{f(z)-f(-z)}\right)^{\alpha}\left(\frac{2\left[\left(z\left(f^{\prime}(z)\right)\right)^{\prime}\right]^{\lambda}}{[f(z)-f(-z)]^{\prime}}\right)^{1-\alpha} \prec \tilde{p}(z)=\frac{1+\tau^{2} z^{2}}{1-\tau z-\tau^{2} z^{2}}
$$

and

$$
\left(\frac{2 w\left(g^{\prime}(w)\right)^{\lambda}}{g(w)-g(-w)}\right)^{\alpha}\left(\frac{2\left[\left(w\left(g^{\prime}(w)\right)\right)^{\prime}\right]^{\lambda}}{[g(w)-g(-w)]^{\prime}}\right)^{1-\alpha} \prec \tilde{p}(w)=\frac{1+\tau^{2} w^{2}}{1-\tau w-\tau^{2} w^{2}}
$$

where $\tau=(1-\sqrt{5}) / 2 \approx-0.618$ where $z, w \in \mathbb{U}$ and $g$ is given by (1.2). 
Specializing the parameter $\lambda=1$ we have the following definitions, respectively:

Definition 2.2. For $0 \leq \alpha \leq 1$, a function $f \in \Sigma$ of the form (1.1) is said to be in the class $\mathcal{P} \mathcal{S}_{s, \Sigma}^{1}(\alpha, \tilde{p}(z)) \equiv \mathcal{M S} \mathcal{L}_{s, \Sigma}(\alpha, \tilde{p}(z))$ if the following subordination hold:

$$
\left(\frac{2 z f^{\prime}(z)}{f(z)-f(-z)}\right)^{\alpha}\left(\frac{2\left(z\left(f^{\prime}(z)\right)\right)^{\prime}}{[f(z)-f(-z)]^{\prime}}\right)^{1-\alpha} \prec \tilde{p}(z)=\frac{1+\tau^{2} z^{2}}{1-\tau z-\tau^{2} z^{2}}
$$

and

$$
\left(\frac{2 w g^{\prime}(w)}{g(w)-g(-w)}\right)^{\alpha}\left(\frac{2\left(w\left(g^{\prime}(w)\right)\right)^{\prime}}{[g(w)-g(-w)]^{\prime}}\right)^{1-\alpha} \prec \tilde{p}(w)=\frac{1+\tau^{2} w^{2}}{1-\tau w-\tau^{2} w^{2}}
$$

where $\tau=(1-\sqrt{5}) / 2 \approx-0.618$ where $z, w \in \mathbb{U}$ and $g$ is given by (1.2).

Further by specializing the parameter $\alpha=1$ and $\alpha=0$ we state the following new classes $\mathcal{S} \mathcal{L}_{s, \Sigma}^{*}(\tilde{p}(z))$ and $\mathcal{K} \mathcal{L}_{s, \Sigma}(\tilde{p}(z))$ respectively.

Definition 2.3. A function $f \in \Sigma$ of the form (1.1) is said to be in the class $\mathcal{P} \mathcal{S} \mathcal{L}_{s, \Sigma}^{1}(1, \tilde{p}(z)) \equiv$ $\mathcal{S L}_{s, \Sigma}^{*}(\tilde{p}(z))$ if the following subordination hold:

$$
\frac{2 z f^{\prime}(z)}{f(z)-f(-z)} \prec \tilde{p}(z)=\frac{1+\tau^{2} z^{2}}{1-\tau z-\tau^{2} z^{2}}
$$

and

$$
\frac{2 w g^{\prime}(w)}{g(w)-g(-w)} \prec \tilde{p}(w)=\frac{1+\tau^{2} w^{2}}{1-\tau w-\tau^{2} w^{2}}
$$

where $\tau=(1-\sqrt{5}) / 2 \approx-0.618$ where $z, w \in \mathbb{U}$ and $g$ is given by (1.2).

Definition 2.4. A function $f \in \Sigma$ of the form (1.1) is said to be in the class $\mathcal{P} \mathcal{S} \mathcal{L}_{s, \Sigma}^{1}(0, \tilde{p}(z)) \equiv$ $\mathcal{K} \mathcal{L}_{s, \Sigma}(\tilde{p}(z))$ if the following subordination hold:

$$
\frac{2\left(z\left(f^{\prime}(z)\right)\right)^{\prime}}{[f(z)-f(-z)]^{\prime}} \prec \tilde{p}(z)=\frac{1+\tau^{2} z^{2}}{1-\tau z-\tau^{2} z^{2}}
$$

and

$$
\frac{2\left(w\left(g^{\prime}(w)\right)\right)^{\prime}}{[g(w)-g(-w)]^{\prime}} \prec \tilde{p}(w)=\frac{1+\tau^{2} w^{2}}{1-\tau w-\tau^{2} w^{2}}
$$

where $\tau=(1-\sqrt{5}) / 2 \approx-0.618$ where $z, w \in \mathbb{U}$ and $g$ is given by (1.2).

Definition 2.5. For $\lambda>0 ; \lambda \neq \frac{1}{3}$, a function $f \in \Sigma$ of the form (1.1) is said to be in the class $\mathcal{P S} \mathcal{L}_{s, \Sigma}^{\lambda}(\tilde{p}(z))$ if the following subordination hold:

$$
\left(\frac{2 z\left(f^{\prime}(z)\right)^{\lambda}}{f(z)-f(-z)}\right) \prec \tilde{p}(z)=\frac{1+\tau^{2} z^{2}}{1-\tau z-\tau^{2} z^{2}}
$$

and

$$
\left(\frac{2 w\left(g^{\prime}(w)\right)^{\lambda}}{g(w)-g(-w)}\right) \prec \tilde{p}(w)=\frac{1+\tau^{2} w^{2}}{1-\tau w-\tau^{2} w^{2}}
$$

where $\tau=(1-\sqrt{5}) / 2 \approx-0.618$ where $z, w \in \mathbb{U}$ and $g$ is given by (1.2). 
Definition 2.6. For $\lambda>0 ; \lambda \neq \frac{1}{3}$, a function $f \in \Sigma$ of the form (1.1) is said to be in the class $\mathcal{G S}_{\mathcal{S}, \Sigma}^{\lambda}(\tilde{p}(z))$ if the following subordination hold:

$$
\left(\frac{2\left[\left(z\left(f^{\prime}(z)\right)\right)^{\prime}\right]^{\lambda}}{[f(z)-f(-z)]^{\prime}}\right) \prec \tilde{p}(z)=\frac{1+\tau^{2} z^{2}}{1-\tau z-\tau^{2} z^{2}}
$$

and

$$
\left(\frac{2\left[\left(w\left(g^{\prime}(w)\right)\right)^{\prime}\right]^{\lambda}}{[g(w)-g(-w)]^{\prime}}\right) \prec \tilde{p}(w)=\frac{1+\tau^{2} w^{2}}{1-\tau w-\tau^{2} w^{2}}
$$

where $\tau=(1-\sqrt{5}) / 2 \approx-0.618$ where $z, w \in \mathbb{U}$ and $g$ is given by (1.2).

In the following theorem we determine the initial Taylor coefficients $\left|a_{2}\right|$ and $\left|a_{3}\right|$ for the function class $\mathcal{P S}_{\mathcal{L}_{s, \Sigma}^{\lambda}}^{\lambda}(\alpha, \tilde{p}(z))$. Later we will reduce these bounds to other classes for special cases.

Theorem 2.7. Let $f$ given by (1.1) be in the class $\mathcal{P} \mathcal{L}_{s, \Sigma}^{\lambda}(\alpha, \tilde{p}(z))$. Then

$$
\left|a_{2}\right| \leq \frac{|\tau|}{\sqrt{4 \lambda^{2}(\alpha-2)^{2}-\left\{10 \lambda^{2}(\alpha-2)^{2}-\lambda-2 \alpha+3\right\} \tau}}
$$

and

$$
\left|a_{3}\right| \leq \frac{2 \lambda|\tau|\left[2 \lambda(\alpha-2)^{2}-\left\{5 \lambda(\alpha-2)^{2}+4-3 \alpha\right\} \tau\right]}{(3 \lambda-1)(3-2 \alpha)\left[4 \lambda^{2}(\alpha-2)^{2}-\left\{10 \lambda^{2}(\alpha-2)^{2}-\lambda-2 \alpha+3\right\} \tau\right]}
$$

where $0 \leq \alpha \leq 1 ; \lambda>0$ and $\lambda \neq \frac{1}{3}$.

Proof. Let $f \in \mathcal{P S}_{\mathcal{L}_{s, \Sigma}^{\lambda}}^{\lambda}(\alpha, \tilde{p}(z))$ and $g=f^{-1}$. Considering (2.7) and (2.8), we have

$$
\left(\frac{2 z\left(f^{\prime}(z)\right)^{\lambda}}{f(z)-f(-z)}\right)^{\alpha}\left(\frac{2\left[\left(z\left(f^{\prime}(z)\right)\right)^{\prime}\right]^{\lambda}}{[f(z)-f(-z)]^{\prime}}\right)^{1-\alpha}=\tilde{p}(u(z))
$$

and

$$
\left(\frac{2 w\left(g^{\prime}(w)\right)^{\lambda}}{g(w)-g(-w)}\right)^{\alpha}\left(\frac{2\left[\left(w\left(g^{\prime}(w)\right)\right)^{\prime}\right]^{\lambda}}{[g(w)-g(-w)]^{\prime}}\right)^{1-\alpha}=\tilde{p}(v(w))
$$

for some Schwarz functions $u$ and $v$ where $\tau=(1-\sqrt{5}) / 2 \approx-0.618$ where $z, w \in \mathbb{U}$ and $g$ is given by (1.2). Since

$$
\begin{aligned}
& \left(\frac{2 z\left[f^{\prime}(z)\right]^{\lambda}}{f(z)-f(-z)}\right)^{\alpha}\left(\frac{2\left[\left(z\left(f^{\prime}(z)\right)\right)^{\prime}\right]^{\lambda}}{[f(z)-f(-z)]^{\prime}}\right)^{1-\alpha} \\
& \quad=1-2 \lambda(\alpha-2) a_{2} z+\left\{\left[2 \lambda^{2}(\alpha-2)^{2}+2 \lambda(3 \alpha-4)\right] a_{2}^{2}+(3 \lambda-1)(3-2 \alpha) a_{3}\right\} z^{2}+\cdots
\end{aligned}
$$

and

$$
\begin{aligned}
& \left(\frac{2 w\left(g^{\prime}(w)\right)^{\lambda}}{g(w)-g(-w)}\right)^{\alpha}\left(\frac{2\left[\left(w\left(g^{\prime}(w)\right)\right)^{\prime}\right]^{\lambda}}{[g(w)-g(-w)]^{\prime}}\right)^{1-\alpha} \\
= & 1+2 \lambda(\alpha-2) a_{2} w+\left\{\left[2 \lambda^{2}(\alpha-2)^{2}+2 \lambda(5-3 \alpha)+2(2 \alpha-3)\right] a_{2}^{2}+(3 \lambda-1)(2 \alpha-3) a_{3}\right\} w^{2}+\cdots .
\end{aligned}
$$


Thus we have

$$
\begin{aligned}
& 1-2 \lambda(\alpha-2) a_{2} z+\left\{\left[2 \lambda^{2}(\alpha-2)^{2}+2 \lambda(3 \alpha-4)\right] a_{2}^{2}+(3 \lambda-1)(3-2 \alpha) a_{3}\right\} z^{2}+\cdots \\
= & 1+\frac{\tilde{p}_{1} c_{1} z}{2}+\left[\frac{1}{2}\left(c_{2}-\frac{c_{1}^{2}}{2}\right) \tilde{p}_{1}+\frac{c_{1}^{2}}{4} \tilde{p}_{2}\right] z^{2} \\
& +\left[\frac{1}{2}\left(c_{3}-c_{1} c_{2}+\frac{c_{1}^{3}}{4}\right) \tilde{p}_{1}+\frac{1}{2} c_{1}\left(c_{2}-\frac{c_{1}^{2}}{2}\right) \tilde{p}_{2}+\frac{c_{1}^{3}}{8} \tilde{p}_{3}\right] z^{3}+\cdots .
\end{aligned}
$$

and

$$
\begin{aligned}
& 1+2 \lambda(\alpha-2) a_{2} w+\left\{\left[2 \lambda^{2}(\alpha-2)^{2}+2 \lambda(5-3 \alpha)+2(2 \alpha-3)\right] a_{2}^{2}+(3 \lambda-1)(2 \alpha-3) a_{3}\right\} w^{2} \\
= & 1+\frac{\tilde{p}_{1} d_{1} w}{2}+\left[\frac{1}{2}\left(d_{2}-\frac{d_{1}^{2}}{2}\right) \tilde{p}_{1}+\frac{d_{1}^{2}}{4} \tilde{p}_{2}\right] w^{2} \\
& +\left[\frac{1}{2}\left(d_{3}-d_{1} d_{2}+\frac{d_{1}^{3}}{4}\right) \tilde{p}_{1}+\frac{1}{2} d_{1}\left(d_{2}-\frac{d_{1}^{2}}{2}\right) \tilde{p}_{2}+\frac{d_{1}^{3}}{8} \tilde{p}_{3}\right] w^{3}+\cdots
\end{aligned}
$$

It follows from (1.5), (2.23) and (2.24) that

$$
\begin{gathered}
-2 \lambda(\alpha-2) a_{2}=\frac{c_{1} \tau}{2} \\
{\left[2 \lambda^{2}(\alpha-2)^{2}+2 \lambda(3 \alpha-4)\right] a_{2}^{2}+(3 \lambda-1)(3-2 \alpha) a_{3}=\frac{1}{2}\left(c_{2}-\frac{c_{1}^{2}}{2}\right) \tau+\frac{3}{4} c_{1}^{2} \tau^{2},}
\end{gathered}
$$

and

$$
\begin{gathered}
2 \lambda(\alpha-2) a_{2}=\frac{d_{1} \tau}{2} \\
{\left[2 \lambda^{2}(\alpha-2)^{2}+2 \lambda(5-3 \alpha)+2(2 \alpha-3)\right] a_{2}^{2}+(3 \lambda-1)(2 \alpha-3) a_{3}=\frac{1}{2}\left(d_{2}-\frac{d_{1}^{2}}{2}\right) \tau+\frac{3}{4} d_{1}^{2} \tau^{2} .}
\end{gathered}
$$

From (2.25) and (2.27), we have

$$
c_{1}=-d_{1}
$$

and

$$
a_{2}^{2}=\frac{\left(c_{1}^{2}+d_{1}^{2}\right)}{32 \lambda^{2}(\alpha-2)^{2}} \tau^{2} .
$$

Now, by summing (2.26) and (2.28), we obtain

$$
\left[4 \lambda^{2}(\alpha-2)^{2}+2(\lambda+2 \alpha-3)\right] a_{2}^{2}=\frac{1}{2}\left(c_{2}+d_{2}\right) \tau-\frac{1}{4}\left(c_{1}^{2}+d_{1}^{2}\right) \tau+\frac{3}{4}\left(c_{1}^{2}+d_{1}^{2}\right) \tau^{2} .
$$

By putting (2.30) in (2.31), we have

$$
2\left[8 \lambda^{2}(\alpha-2)^{2}-\left\{20 \lambda^{2}(\alpha-2)^{2}-2(\lambda+2 \alpha-3)\right\} \tau\right] a_{2}^{2}=\left(c_{2}+d_{2}\right) \tau^{2} .
$$

Therefore, using Lemma 1.3 we obtain

$$
\left|a_{2}\right| \leq \frac{|\tau|}{\sqrt{4 \lambda^{2}(\alpha-2)^{2}-\left\{10 \lambda^{2}(\alpha-2)^{2}-\lambda-2 \alpha+3\right\} \tau}} .
$$

Now, so as to find the bound on $\left|a_{3}\right|$, let's subtract from (2.26) and (2.28). So, we find

$$
2(3 \lambda-1)(3-2 \alpha) a_{3}-2(3 \lambda-1)(3-2 \alpha) a_{2}^{2}=\frac{1}{2}\left(c_{2}-d_{2}\right) \tau .
$$


Hence, we get

$$
2(3 \lambda-1)(3-2 \alpha)\left|a_{3}\right| \leq 2|\tau|+2(3 \lambda-1)(3-2 \alpha)\left|a_{2}\right|^{2} .
$$

Then, in view of (2.33), we obtain

$$
\left|a_{3}\right| \leq \frac{2 \lambda|\tau|\left[2 \lambda(\alpha-2)^{2}-\left\{5 \lambda(\alpha-2)^{2}+4-3 \alpha\right\} \tau\right]}{(3 \lambda-1)(3-2 \alpha)\left[4 \lambda^{2}(\alpha-2)^{2}-\left\{10 \lambda^{2}(\alpha-2)^{2}-\lambda-2 \alpha+3\right\} \tau\right]} .
$$

If we can take the parameter $\lambda=1$ in the above theorem, we have the following the initial Taylor coefficients $\left|a_{2}\right|$ and $\left|a_{3}\right|$ for the function classes $\mathcal{M S}_{\mathcal{L}, \Sigma}(\alpha, \tilde{p}(z))$.

Corollary 2.8. Let $f$ given by (1.1) be in the class $\mathcal{M S L}_{s, \Sigma}(\alpha, \tilde{p}(z))$. Then

$$
\left|a_{2}\right| \leq \frac{|\tau|}{\sqrt{4(\alpha-2)^{2}-2\left(5 \alpha^{2}-21 \alpha+21\right) \tau}}
$$

and

$$
\left|a_{3}\right| \leq \frac{|\tau|\left[2(\alpha-2)^{2}-\left\{5 \alpha^{2}-23 \alpha+24\right\} \tau\right]}{(3-2 \alpha)\left[4(\alpha-2)^{2}-\left\{10 \alpha^{2}-42 \alpha+42\right\} \tau\right]}
$$

Further by taking $\alpha=1$ and $\alpha=0$ and $\tau=-0.618$ in Corollary 2.8, we have the following improved initial Taylor coefficients $\left|a_{2}\right|$ and $\left|a_{3}\right|$ for the function classes $\mathcal{S L}_{s, \Sigma}^{*}(\tilde{p}(z))$ and $\mathcal{K} \mathcal{L}_{s, \Sigma}(\tilde{p}(z))$ respectively.

Corollary 2.9. Let $f$ given by (1.1) be in the class $\mathcal{S L}_{s, \Sigma}^{*}(\tilde{p}(z))$. Then

$$
\left|a_{2}\right| \leq \frac{|\tau|}{\sqrt{4-10 \tau}} \simeq 0.19369
$$

and

$$
\left|a_{3}\right| \leq \frac{|\tau|(1-3 \tau)}{2-5 \tau} \simeq 0.3465 .
$$

Corollary 2.10. Let $f$ given by (1.1) be in the class $\mathcal{K} \mathcal{L}_{s, \Sigma}(\tilde{p}(z))$. Then

$$
\left|a_{2}\right| \leq \frac{|\tau|}{\sqrt{16-42 \tau}} \simeq 0.0954
$$

and

$$
\left|a_{3}\right| \leq \frac{4|\tau|(1-3 \tau)}{3(8-21 \tau)} \simeq 0.17647
$$

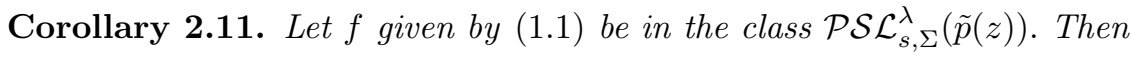

$$
\left|a_{2}\right| \leq \frac{|\tau|}{\sqrt{4 \lambda^{2}-\left\{10 \lambda^{2}-\lambda+1\right\} \tau}}
$$

and

$$
\left|a_{3}\right| \leq \frac{2 \lambda|\tau|[2 \lambda-\{5 \lambda+1\} \tau]}{(3 \lambda-1)\left[4 \lambda^{2}-\left\{10 \lambda^{2}-\lambda+1\right\} \tau\right]}
$$

where $\lambda>0$ and $\lambda \neq \frac{1}{3}$. 
Corollary 2.12. Let $f$ given by (1.1) be in the class $\mathcal{G S} \mathcal{L}_{s, \Sigma}^{\lambda}(\tilde{p}(z))$. Then

$$
\left|a_{2}\right| \leq \frac{|\tau|}{\sqrt{16 \lambda^{2}-\left\{40 \lambda^{2}-\lambda+3\right\} \tau}}
$$

and

$$
\left|a_{3}\right| \leq \frac{2 \lambda|\tau|[8 \lambda-\{20 \lambda+4\} \tau]}{3(3 \lambda-1)\left[16 \lambda^{2}-\left\{40 \lambda^{2}-\lambda+3\right\} \tau\right]}
$$

where $\lambda>0$ and $\lambda \neq \frac{1}{3}$.

\section{$3 \quad$ Fekete-Szegö inequality for the function class $\mathcal{P S} \mathcal{L}_{s, \Sigma}^{\lambda}(\alpha, \tilde{p}(z))$}

Fekete and Szegö [7] introduced the generalized functional $\left|a_{3}-\mu a_{2}^{2}\right|$, where $\mu$ is some real number. Due to Zaprawa [19], in the following theorem we determine the Fekete-Szegö functional for $f \in$ $\mathcal{P} \mathcal{S}_{s, \Sigma}^{\lambda}(\alpha, \tilde{p}(z))$.

Theorem 3.1. Let $\lambda \in \mathbb{R}$ with $\lambda>\frac{1}{3}$ and let $f$ given by (1.1) be in the class $\mathcal{P S}_{\mathcal{L}_{s, \Sigma}^{\lambda}}(\alpha, \tilde{p}(z))$ and $\mu \in \mathbb{R}$. Then we have

$$
\left|a_{3}-\mu a_{2}^{2}\right| \leq\left\{\begin{array}{lr}
\frac{|\tau|}{4(3 \lambda-1)(3-2 \alpha)}, & 0 \leq|h(\mu)| \leq \frac{|\tau|}{4(3 \lambda-1)(3-2 \alpha)} \\
4|h(\mu)|, & |h(\mu)| \geq \frac{|\tau|}{4(3 \lambda-1)(3-2 \alpha)}
\end{array}\right.
$$

where

$$
h(\mu)=\frac{(1-\mu) \tau^{2}}{4\left[4 \lambda^{2}(\alpha-2)^{2}-\left\{10 \lambda^{2}(\alpha-2)^{2}-\lambda-2 \alpha+3\right\} \tau\right]} .
$$

Proof. From (2.32) and (2.34) we obtain

$$
\begin{aligned}
a_{3}-\mu a_{2}^{2} & =\frac{(1-\mu)\left(c_{2}+d_{2}\right) \tau^{2}}{4\left[4 \lambda^{2}(\alpha-2)^{2}-\left\{10 \lambda^{2}(\alpha-2)^{2}-\lambda-2 \alpha+3\right\} \tau\right]}+\frac{\tau\left(c_{2}-d_{2}\right)}{4(3 \lambda-1)(3-2 \alpha)} \\
& =\left(\frac{(1-\mu) \tau^{2}}{4\left[4 \lambda^{2}(\alpha-2)^{2}-\left\{10 \lambda^{2}(\alpha-2)^{2}-\lambda-2 \alpha+3\right\} \tau\right]}+\frac{\tau}{4(3 \lambda-1)(3-2 \alpha)}\right) c_{2} \\
& +\left(\frac{(1-\mu) \tau^{2}}{4\left[4 \lambda^{2}(\alpha-2)^{2}-\left\{10 \lambda^{2}(\alpha-2)^{2}-\lambda-2 \alpha+3\right\} \tau\right]}-\frac{\tau}{4(3 \lambda-1)(3-2 \alpha)}\right) d_{2} .
\end{aligned}
$$

So we have

$$
a_{3}-\mu a_{2}^{2}=\left(h(\mu)+\frac{\tau}{4(3 \lambda-1)(3-2 \alpha)}\right) c_{2}+\left(h(\mu)-\frac{\tau}{4(3 \lambda-1)(3-2 \alpha)}\right) d_{2}
$$

where

$$
h(\mu)=\frac{(1-\mu) \tau^{2}}{4\left[4 \lambda^{2}(\alpha-2)^{2}-\left\{10 \lambda^{2}(\alpha-2)^{2}-\lambda-2 \alpha+3\right\} \tau\right]} .
$$

Then, by taking modulus of (3.2), we conclude that 


$$
\left|a_{3}-\mu a_{2}^{2}\right| \leq\left\{\begin{array}{lr}
\frac{|\tau|}{4(3 \lambda-1)(3-2 \alpha)}, & 0 \leq|h(\mu)| \leq \frac{|\tau|}{4(3 \lambda-1)(3-2 \alpha)} \\
4|h(\mu)|, & |h(\mu)| \geq \frac{|\tau|}{4(3 \lambda-1)(3-2 \alpha)}
\end{array}\right.
$$

Taking $\mu=1$, we have the following corollary.

Corollary 3.2. If $f \in \mathcal{P} \mathcal{S} \mathcal{L}_{s, \Sigma}^{\lambda}(\alpha, \tilde{p}(z))$, then

$$
\left|a_{3}-a_{2}^{2}\right| \leq \frac{|\tau|}{4(3 \lambda-1)(3-2 \alpha)} .
$$

If we can take the parameter $\lambda=1$ in Theorem 3.1, we can state the following:

Corollary 3.3. Let $f$ given by (1.1) be in the class $\mathcal{M S}_{s, \Sigma}(\alpha, \tilde{p}(z))$ and $\mu \in \mathbb{R}$. Then we have

$$
\left|a_{3}-\mu a_{2}^{2}\right| \leq\left\{\begin{array}{lr}
\frac{|\tau|}{8(3-2 \alpha)}, & 0 \leq|h(\mu)| \leq \frac{|\tau|}{8(3-2 \alpha)} \\
4|h(\mu)|, & |h(\mu)| \geq \frac{|\tau|}{8(3-2 \alpha)}
\end{array}\right.
$$

where

$$
h(\mu)=\frac{(1-\mu) \tau^{2}}{4\left[4(\alpha-2)^{2}-\left\{10(\alpha-2)^{2}-2 \alpha+2\right\} \tau\right]} .
$$

Further by fixing $\lambda=1$ taking $\alpha=1$ and $\alpha=0$ in the above corollary, we have the following the Fekete-Szegö inequalities for the function classes $\mathcal{S L}_{s, \Sigma}^{*}(\tilde{p}(z))$ and $\mathcal{K} \mathcal{L}_{s, \Sigma}(\tilde{p}(z))$, respectively.

Corollary 3.4. Let $f$ given by (1.1) be in the class $\mathcal{S L}_{s, \Sigma}^{*}(\tilde{p}(z))$ and $\mu \in \mathbb{R}$. Then we have

$$
\left|a_{3}-\mu a_{2}^{2}\right| \leq\left\{\begin{array}{lr}
\frac{|\tau|}{24}, & 0 \leq|h(\mu)| \leq \frac{|\tau|}{24} \\
4|h(\mu)|, & |h(\mu)| \geq \frac{|\tau|}{24}
\end{array}\right.
$$

where $h(\mu)=\frac{(1-\mu) \tau^{2}}{8[2-5 \tau]}$.

Corollary 3.5. Let $f$ given by (1.1) be in the class $\mathcal{K} \mathcal{L}_{s, \Sigma}(\tilde{p}(z))$ and $\mu \in \mathbb{R}$. Then we have

$$
\left|a_{3}-\mu a_{2}^{2}\right| \leq\left\{\begin{array}{lrl}
\frac{|\tau|}{8}, & 0 \leq|h(\mu)| & \leq \frac{|\tau|}{8} \\
4|h(\mu)|, & |h(\mu)| & \geq \frac{|\tau|}{8}
\end{array}\right.
$$

where $h(\mu)=\frac{(1-\mu) \tau^{2}}{8[8-21 \tau]}$.

By assuming $\lambda \in \mathbb{R} ; \lambda>\frac{1}{3}$ and taking $\alpha=1$ and $\alpha=0$ we have the following the Fekete-Szegö inequalities for the function classes $\mathcal{P} \mathcal{S L}_{s, \Sigma}^{\lambda}(\tilde{p}(z))$ and $\mathcal{G S}_{s, \Sigma}^{\lambda}(\tilde{p}(z))$, respectively. 
Corollary 3.6. Let $\lambda \in \mathbb{R}$ with $\lambda>\frac{1}{3}$ and let $f$ given by (1.1) be in the class $\mathcal{P S}_{\mathcal{L}}^{\lambda},(\tilde{p}(z))$ and $\mu \in \mathbb{R}$. Then we have

$$
\left|a_{3}-\mu a_{2}^{2}\right| \leq\left\{\begin{array}{lr}
\frac{|\tau|}{4(3 \lambda-1)}, & 0 \leq|h(\mu)| \leq \frac{|\tau|}{4(3 \lambda-1)} \\
4|h(\mu)|, & |h(\mu)| \geq \frac{|\tau|}{4(3 \lambda-1)}
\end{array}\right.
$$

where

$$
h(\mu)=\frac{(1-\mu) \tau^{2}}{4\left[4 \lambda^{2}-\left\{10 \lambda^{2}-\lambda+1\right\} \tau\right]} .
$$

Corollary 3.7. Let $\lambda \in \mathbb{R}$ with $\lambda>\frac{1}{3}$ and let $f$ given by (1.1) be in the class $\mathcal{G S}_{\mathcal{L}_{s, \Sigma}^{\lambda}}^{\lambda}(\tilde{p}(z))$ and $\mu \in \mathbb{R}$. Then we have

$$
\left|a_{3}-\mu a_{2}^{2}\right| \leq\left\{\begin{array}{lr}
\frac{|\tau|}{12(3 \lambda-1)}, & 0 \leq|h(\mu)| \leq \frac{|\tau|}{12(3 \lambda-1)} \\
4|h(\mu)|, & |h(\mu)| \geq \frac{|\tau|}{12(3 \lambda-1)}
\end{array}\right.
$$

where

$$
h(\mu)=\frac{(1-\mu) \tau^{2}}{4\left[16 \lambda^{2}-\left\{40 \lambda^{2}-\lambda+3\right\} \tau\right]} .
$$

\section{Conclusions}

Our motivation is to get many interesting and fruitful usages of a wide variety of Fibonacci numbers in Geometric Function Theory. By defining a subclass $\lambda$-bi-pseudo-starlike functions with respect to symmetric points of $\Sigma$ related to shell-like curves connected with Fibonacci numbers we were able to unify and extend the various classes of analytic bi-univalent function, and new extensions were discussed in detail. Further, by specializing $\alpha=0$ and $\alpha=1$ and $\tau=-0.618$ we have attempted at the discretization of some of the new and well-known results. Our main results are new and better improvement to initial Taylor-Maclaurin coefficients $\left|a_{2}\right|$ and $\left|a_{3}\right|$.

\section{Acknowledgements}

The authors thank the referees of this paper for their insightful suggestions and corrections to improve the paper in present form. 


\section{References}

[1] K. O. Babalola, "On $\lambda$-pseudo-starlike functions", J. Class. Anal., vol. 3, no. 2, pp. 137-147, 2013.

[2] D. A. Brannan, J. Clunie and W. E. Kirwan, "Coefficient estimates for a class of star-like functions", Canad. J. Math., vol. 22, no. 3, pp. 476-485, 1970.

[3] D. A. Brannan and T. S. Taha, "On some classes of bi-univalent functions", Studia Univ. Babes-Bolyai Math., vol. 31, no. 2, pp. 70-77, 1986.

[4] P. L. Duren, Univalent Functions, Grundlehren der Mathematischen Wissenschaften, Band 259, New York, Berlin, Heidelberg and Tokyo: Springer-Verlag, 1983.

[5] S. Joshi, S. Joshi and H. Pawar, "On some subclasses of bi-univalent functions associated with pseudo-starlike function", J. Egyptian Math. Soc., vol. 24, no. 4, pp. 522-525, 2016.

[6] J. Dziok, R. K. Raina and J. Sokół, "On $\alpha$-convex functions related to a shell-like curve connected with Fibonacci numbers", Appl. Math. Comput., vol. 218, no. 3, pp. 996-1002, 2011.

[7] M. Fekete and G. Szegö, "Eine Bemerkung über ungerade Schlichte Funktionen", J. London Math. Soc., vol. 8, no. 2, pp. 85-89, 1933.

[8] S. S. Miller and P. T. Mocanu Differential Subordinations Theory and Applications, Series of Monographs and Text Books in Pure and Applied Mathematics, vol. 225, New York: Marcel Dekker, 2000.

[9] M. Lewin, "On a coefficient problem for bi-univalent functions", Proc. Amer. Math. Soc., vol. 18, pp. 63-68, 1967.

[10] Ch. Pommerenke, Univalent Functions, Math. Math, Lehrbucher, Vandenhoeck and Ruprecht, Göttingen, 1975.

[11] R. K. Raina and J. Sokół, "Fekete-Szegö problem for some starlike functions related to shelllike curves", Math. Slovaca, vol. 66, no. 1, pp. 135-140, 2016.

[12] V. Ravichandran, "Starlike and convex functions with respect to conjugate points", Acta Math. Acad. Paedagog. Nyházi. (N.S.), vol. 20, no. 1, pp. 31-37, 2004.

[13] K. Sakaguchi, "On a certain univalent mapping", J. Math. Soc. Japan, vol. 11, no. 1, pp. $72-75,1959$.

[14] J. Sokół, "On starlike functions connected with Fibonacci numbers", Zeszyty Nauk. Politech. Rzeszowskiej Mat, vol. 23, pp. 111-116, 1999. 
[15] H. M. Srivastava, A. K. Mishra and P. Gochhayat, "Certain subclasses of analytic and biunivalent functions", Appl. Math. Lett., vol. 23, no. 10, pp. 1188-1192, 2010.

[16] Q.-H. Xu, Y.-C. Gui and H. M. Srivastava, "Coefficient estimates for a certain subclass of analytic and bi-univalent functions", Appl. Math. Lett., vol. 25, no. 6, pp. 990-994, 2012.

[17] X.-F. Li and A.-P. Wang, "Two new subclasses of bi-univalent functions", Int. Math. Forum, vol. 7, no. 30, pp. 1495-1504, 2012.

[18] G. Wang, C. Y. Gao and S. M. Yuan, "On certain subclasses of close-to-convex and quasiconvex functions with respect to $k$-symmetric points", J. Math. Anal. Appl., vol. 322, no. 1, pp. 97-106, 2006.

[19] P. Zaprawa, "On the Fekete-Szegö problem for classes of bi-univalent functions", Bull. Belg. Math. Soc. Simon Stevin, vol. 21, no. 1, pp. 169-178, 2014. 